\title{
New perspectives for acute stroke treatment: the role of mechanical thrombectomy
}

\author{
Agnieszka Slowik \\ Department of Neurology, Medical College Jagiellonian University, Krakow, Poland
}

Postep Kardiol Inter 2014; 10, 3 (37): 145-146 DOI: $10.5114 /$ pwki.2014.45138

Stroke is the third leading cause of death and a major cause of disability in adults. In Poland, up to 80,000 people suffer from stroke each year; $25 \%$ die within 90 days, and those who survive need support in everyday life.

Acute stroke treatment with IV rt-PA, routinely available since the 1990s, can be utilised in only a small percentage of stroke victims. IV rt-PA gives a $30 \%$ increase in the number of patients who recover completely. The low percentage of patients who can be treated with IV rt-PA is due to its narrow therapeutic window (4.5 h). During that timeframe, not only must a detailed interview be conducted and a neurological examination be performed, but also brain neuroimaging and several blood tests must be obtained. To complete all of these procedures in such a short timeframe requires perfect organisation and the close collaboration of all parties involved in the treatment process. In Poland, treatment with IV rt-PA is performed in stroke units that are typically divisions of neurology departments.

In the last few years, leading European and American stroke centres have introduced endovascular treatment - mechanical thrombectomy and, to a much lesser extent, pharmacological treatment by intra-arterial (IA) rtPA. Pharmacological IA treatment can only be performed following consultation with an ethics committee, since the efficacy of these interventional treatments has not been confirmed in large clinical trials. It should be noted that mechanical thrombectomy can be used up to $8 \mathrm{~h}$ following onset of symptoms for strokes located in the anterior circulation, and in some specific cases even up to $24 \mathrm{~h}$ later. This wider treatment window allows us to employ this interventional treatment for many more victims of acute stroke. Information concerning the current indication for endovascular treatment of acute stroke is available in European (European Stroke Organisation) and American (American Heart Association/American Stroke Association) guidelines.
The first device for mechanical thrombectomy in acute stroke, MERCI (Concentric Medical), was approved by the FDA in the US 10 years ago. The second device, Penumbra (Penumbra, Alameda, CA, USA), was approved several years later. Although both devices increased significantly the percentage of brain vessels that were re-canalised, the percentage of patients who recovered completely was still less than $25 \%$.

Stent retrievers, a new generation of devices for mechanical thrombectomy, have recently been introduced and are more efficacious and safer than the older devices. In 2012 the FDA and the EMA registered two stent retrievers: SOLITAIRE (Solitaire.ev3.Irvine, CA, USA) and TREVO (Trevo; Stryker, Kalamazoo, MI, USA). Their significant advantage as compared with MERCI was shown in two important clinical studies: the Swift (SOLITAIRE) trial and the Trevo 2 trial (TREVO), published in "Lancet" in 2012 [1, 2]. Both studies showed a very high percentage of recanalisation: $83 \%$ (Solitaire) and 89\% (Trevo). Fifty-eight percent of patients treated with Solitaire and $55 \%$ of those treated with Trevo recovered completely. Most importantly, the percentage of patients who developed brain haemorrhages due to treatment with these devices was low: $2 \%$ in patients treated with Solitaire and $6.8 \%$ in patients treated with Trevo. The efficacy of both retrievers was later confirmed in national registries. An increasing number of stent retrievers with new modifications is now available, including CATCH+ (BALT), pREset (Phenox), ASPERIO (Acandis), and Revive SE (Codman).

In 2013, optimism related to endovascular treatment of acute stroke was considerably tempered, since the results of three negative clinical studies that compared mechanical thrombectomy with standardised treatment were published in NEJM ("New England Medical Journal”). The first study, "Endovascular Therapy after intravenous t-PA versus t-PA alone for stroke (IMSIII)" [3], included 656 acute stroke patients. Two hundred and twenty-two

Corresponding author:

Prof. Agnieszka Slowik MD, PhD, Department of Neurology, Medical College Jagiellonian University, 3 Botaniczna St, 31-503 Krakow, Poland, phone: +48 12 4248694, e-mail: agnieszka.slowik@uj.edu.pl

Received: 31.08 .2014 , accepted: 1.09.2014. 
patients were treated with IV rt-PA alone within $3 \mathrm{~h}$ after stroke onset, while the remaining patients received endovascular treatment in addition to IV-rt-PA. The second study, "A Trial of Imaging selection and endovascular treatment for ischaemic stroke" [4], was performed in 118 patients recruited within $8 \mathrm{~h}$ after the onset of large-vessel anterior-circulation strokes. Seventy patients received mechanical thrombectomy while 57 patients received standard treatment. The third study, "Endovascular Treatment of acute ischaemic stroke: SYNTHESIS EXPANSION" [5], included 362 patients. Treatment was initiated within $4.5 \mathrm{~h}$ following stroke onset: $50 \%$ received endovascular treatment (IA rt-PA and/or mechanical thrombectomy), and 50\% received IV rt-PA only. The publication of these papers precipitated a lively discussion in the literature, including the NEJM, and resulted in several objections to the study protocols, including the slow recruitment of patients, the lack of information concerning anaesthesia, the lack of routine brain vessel imaging that would have localised the thrombus prior to the procedure, and most importantly, the use of primarily older devices for mechanical thrombectomy.

Although these three studies did not show an advantage for endovascular treatment over standard therapy in acute stroke, detailed analysis of all clinical studies and registries published so far, as well as several personal communications and comments published in leading journals, allows one to conclude the following: 1) the new generation of devices for mechanical thrombectomy (stent retrievers) are more efficacious than older devices such as MERCI (better recanalisation, higher percentage of patients who recovered completely), and these devices are now the most commonly used ones for mechanical thrombectomy in acute stroke; 2) endovascular treatment is efficacious only in a small percentage of acute stroke patients, i.e. patients with more severe stroke due to occlusion of $\mathrm{M} 1$ or $\mathrm{M} 2$ of the middle cerebral artery or the basilar or vertebral arteries (IV rt-PA is much less efficacious in these cases); and 3) before deciding to proceed with endovascular treatment, patients should have imaging of brain vessels performed to localise the thrombus and to exclude arterio-venous malformations or carotid artery stenosis.

At present, several clinical trials to evaluate the significance of endovascular treatment as compared with standardised therapy are in progress (http://www.snisonline.org/stroketrials). Unfortunately, recruitment for these trials is proceeding slowly and the results are expected no earlier than within the next few years. This is because several new devices for mechanical thrombectomy in acute stroke have already been approved by the FDA and the EMA, and patients prefer treatment with mechanical thrombectomy in a clinical setting rather than by participating in clinical trials.

In everyday practice, many countries require that all stroke patients treated with mechanical thrombectomy be entered into standard registries, for example the SITS
Thrombectomy Registry, which includes patients from several European countries. This approach is very helpful in evaluating the efficacy and complications of these rare procedures.

In the USA, endovascular treatment in acute stroke is performed in highly specialised Comprehensive Stroke Centres. In 2005, the Brain Attack Coalition published the requirements for such centres [6]. According to this document, Comprehensive Stroke Centres should employ several high quality experts in stroke and subarachnoid haemorrhage, including neurologists, radiologists, vascular surgeons, neurosurgeons, and anaesthesiologists. Each Comprehensive Stroke Centre must have access to MRI/MRA, CTA, DSA, and TCD and round-the-clock (24/7) access to interventional treatment.

Epidemiological data from the USA indicate that no more than $2 \%$ of stroke victims undergo endovascular treatment (compared with $70 \%$ of patients with acute coronary disease who undergo endovascular treatment at present).

In the USA, there is a need for approximately $200 \mathrm{Com}$ prehensive Stroke Centres and 600-800 interventional radiologists (three specialists for each centre). In Poland, the estimated number of patients who potentially fulfil criteria for endovascular treatment varies from 1500-2000. The estimated number of Comprehensive Stroke Centres that are required varies from $15-20$.

In Poland, the first centre that fulfils the criteria for a Comprehensive Stroke Centre and has introduced roundthe-clock access for endovascular acute stroke treatment is the Centre for Interventional Therapy of Acute Stroke at the University Hospital in Krakow. So far, 40 acute stroke patients were treated with mechanical thrombectomy and all are followed according to the protocol approved by the local ethics committee. The experience of the Krakow Comprehensive Stroke Centre may help to establish other Comprehensive Stroke Centres in Poland.

\section{References}

1. NogueiraRG, Lutsep HL, Gupta R, et al. Trevo versus Merci retrievers for thrombectomy revascularisation of large vessel occlusions in acute ischaemic stroke (TREVO 2): a randomised trial. Lancet 2012; 380: 1231-40.

2. Saver JL, Jahan R, Levy El, et al. Solitaire flow restoration device versus the Merci Retriever in patients with acute ischaemic stroke (SWIFT): a randomised, parallel-group, non-inferiority trial. Lancet 2012; 380: 1241-9.

3. Broderick JP, PaleschYY, Demchuk AM, et al. Endovascular therapy after intravenous t-PA versus t-PA alone for stroke. N Engl J Med 2013; 368: 893-903.

4. Kidwell CS, Jahan R, Gornbein J, et al. A trial of imaging selection and endovascular treatment for ischemic stroke. N Engl J Med 2013; 368: 914-23.

5. Ciccone A, Valvassori L, Nichelatti $M$, et al. Endovascular treatment for acute ischemic stroke. N Engl J Med 2013; 368: 904-13.

6. Alberts MJ, Latchaw RE, Selman WR, et al. Recommendations for comprehensive stroke centers: a consensus statement from the Brain Attack Coalition. Stroke 2005; 36: 1597-616. 